PHYSICAL REVIEW D 92, 079901(E) (2015)

\title{
Publisher's Note: Measurement of the proton-air cross section with Telescope Array's Middle Drum detector and surface array in hybrid mode [Phys. Rev. D 92, 032007 (2015)]
}

R. U. Abbasi, M. Abe, T. Abu-Zayyad, M. Allen, R. Azuma, E. Barcikowski, J. W. Belz, D. R. Bergman, S. A. Blake, R. Cady, M. J. Chae, B. G. Cheon, J. Chiba, M. Chikawa, W. R. Cho, T. Fujii, M. Fukushima, T. Goto, W. Hanlon, Y. Hayashi, N. Hayashida, K. Hibino, K. Honda, D. Ikeda, N. Inoue, T. Ishii, R. Ishimori, H. Ito, D. Ivanov, C. C. H. Jui, K. Kadota, F. Kakimoto, O. Kalashev, K. Kasahara, H. Kawai, S. Kawakami, S. Kawana, K. Kawata, E. Kido, H. B. Kim, J. H. Kim, J. H. Kim, S. Kitamura, Y. Kitamura, V. Kuzmin, Y. J. Kwon, J. Lan, S. I. Lim, J. P. Lundquist, K. Machida, K. Martens, T. Matsuda, T. Matsuyama, J. N. Matthews, M. Minamino, Y. Mukai, I. Myers, K. Nagasawa, S. Nagataki, T. Nakamura, T. Nonaka, A. Nozato, S. Ogio, J. Ogura, M. Ohnishi, H. Ohoka, K. Oki, T. Okuda, M. Ono, A. Oshima, S. Ozawa, I. H. Park, M. S. Pshirkov, D. C. Rodriguez, G. Rubtsov, D. Ryu, H. Sagawa, N. Sakurai, L. M. Scott, P. D. Shah, F. Shibata, T. Shibata, H. Shimodaira, B. K. Shin, H. S. Shin, J. D. Smith, P. Sokolsky, R. W. Springer, B. T. Stokes, S. R. Stratton, T. A. Stroman, T. Suzawa, M. Takamura, M. Takeda, R. Takeishi, A. Taketa, M. Takita, Y. Tameda, H. Tanaka, K. Tanaka, M. Tanaka, S. B. Thomas, G. B. Thomson, P. Tinyakov, I. Tkachev, H. Tokuno, T. Tomida, S. Troitsky, Y. Tsunesada, K. Tsutsumi, Y. Uchihori, S. Udo, F. Urban, G. Vasiloff, T. Wong, R. Yamane, H. Yamaoka, K. Yamazaki, J. Yang, K. Yashiro, Y. Yoneda, S. Yoshida, H. Yoshii, R. Zollinger, and Z. Zundel

(Telescope Array Collaboration)

(Received 22 September 2015; published 2 October 2015)

DOI: 10.1103/PhysRevD.92.079901

PACS numbers: 13.85.Lg, 13.85.Tp, 99.10.Fg

This paper was published online on 25 August 2015 with an omission of an author footnote. R. U. Abbasi's footnote should read as “rasha@ cosmic.utag.edu.” The paper has been corrected as of 14 September 2015. The author footnote is not present in the printed version of the journal. 